International Journal of Engineering \& Technology, $7(2.33)(2018) 397-400$
International Journal of Engineering \& Technology
Website: $w w w . s c i e n c e p u b c o . c o m / i n d e x . p h p / I J E T$
Research paper

\title{
3D interactive visualization using image color distribution
}

\author{
Chaelin Lee ${ }^{1}$, Sanghyun Seo ${ }^{1 *}$ \\ ${ }^{1}$ Div. of Media-software, Sungkyul University, 53 Sungkyuldaehak-ro, Manan-GU, Anyang, 14097, Rep. of Korea \\ *Corresponding author E-mail: shseo75@gmail.com
}

\begin{abstract}
Background/Objectives: Technologies related to image processing such as transforming the atmosphere of images or adding effects to images have been making rapid progress owing to the recent advancement of media.

Methods/Statistical analysis: We need to devise methods to easily identify color composition and distribution in 3D space. This study introduces a method of visualizing the color distribution in 3D using standard color models so that the distribution pattern of color information in images can be easily understood.

Findings: The distribution of colors that make up these images provides people with various stimuli and cognitive information. In order to convert images according to the user's intention in image manipulation research, the process of analyzing the images is very important, yet it is also significant to confirm that they have been converted as intended.
\end{abstract}

Improvements/Applications: Our proposed method enables the user to intuitively understand and recognize color information of image.

Keywords: Information Visualization; Color Distribution; 3D Visualization; Color Model; Info-Graphic

\section{Introduction}

Research on image processing has been actively under way due to the advancement of media. Various types of information such as background color, illumination, position, etc., are included in images. Currently, techniques such as recreating images with different atmospheres from original ones or adding effects to them by transforming these types of information are being introduced. In order to transform images according to the user's intention, the process of analyzing and converting them is very important, yet it is also significant to confirm that they have been converted as intended. One way to verify that images have been converted as intended is to use all the colors as frequency band graphs according to the wavelength. However, because this is difficult to achieve, the most common method is to use color models in color analysis. Nevertheless, it is not easy to determine if the images have been converted properly for the user through letters or numbers because they are difficult to understand. To address this issue, we will implement a method for quickly recognizing the results of image conversions using the most appropriate out of the various information visualization methods.

Information visualization is the method of expressing information as images to help the user who has access to this information to understand it quickly [1-2]. In the past, we used charts to understand the detailed flow of analysis results of various kinds of information or task procedures. In addition, they were represented in graphs so that the information sizes could be easily compared and the changes could be easily understood at a glance. Of those methods, the histogram is the most frequently used method to visualize image analysis information. The color histogram is a method of representing output values from analyzing the color of images in a bar graph on a 2dimensional plane. This method uses 2-dimensional Gaussian coordinates similar to other 2-dimensional graphs. The horizontal Xaxis represents the color ranging from 0 to 255 , and the vertical Yaxis represents the amount of each color. In addition, R, G, and B channels of an image are separated into a single channel, and then the results are visualized [3].

Figure 1 shows a histogram for each channel (R, G, B) in the RGB color model. The distribution for each channel is clearly recognized. However, it is difficult to confirm the distribution of the final color information resulting from the integration of each channel, which makes it difficult to confirm the relationship between colors, which means that only approximate information can be confirmed, a drawback of the method.

These days, the advancement of media allows information, data, and knowledge to be visually represented. Thus, various representations such as motion, storytelling interaction, dynamic, and 3-dimensional graphics have been developed and the traditional infographics that can transmit information quickly and easily [4-5].

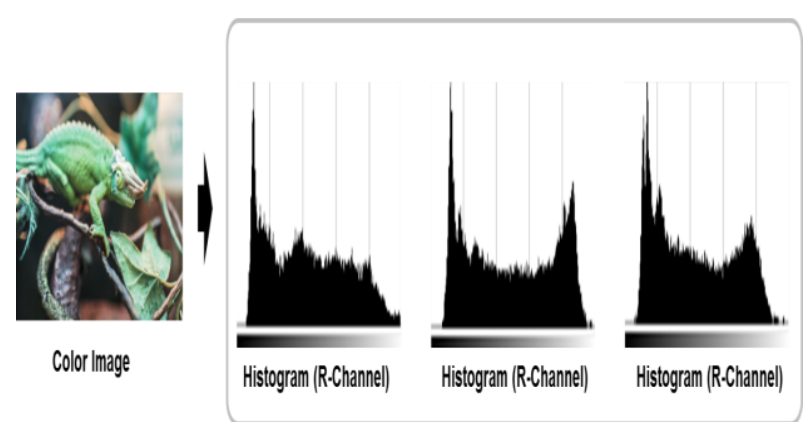

Fig. 1: Examples of Color Histograms.

In this study, we will visualize the results of the analyses of images through a three-dimensional space structure among information visualization methods so that the user can quickly recognize them. In addition, we aim to achieve visualization as intended by the user by providing visualization control parameters that can control standard color models (RGB, HSV, and Lab) and resolutions. Various tech- 
niques such as color space analysis, clustering technique, and maximization of 3-dimensional space utilization are included to visualize the colors of images in a 3-dimensional space.

Currently, there are various color models that represent the color of images. Of them, RGB, HSV, Lab, and etc., are the commonly used color models. These different color models are used for different purposes. First, the RGB color model consists of the three primary colors of red, green and blue, and the spectral components of each color are additionally mixed together to produce different colors. The HSV color model refers to a kind of color space based on perception. It expresses the Munsell coordinate system by modeling hue, saturation and value as the 3-dimensional space. Lastly, the Lab color model is a color model that is similar to a human color perception system. With this model, the difference in colors we perceive is similar to the difference of distance in a color space.

It is important for the user to intuitively know which data needs to be highlighted in order to visualize data in a 3-dimensional space. A study on 'Methods to maximize 3-dimensional space use in information visualization' suggested that when a large amount of data is represented in a certain graphical space, it should be expressed as much as possible in the graphical space and certain data could be highlighted selectively [6]. There are also analytical studies on the complexity of information visualization. A study 'Efficient scene description method with the rendering complexity rate functionality' proposed that relatively light media objects were rendered first by referring to a 'rendering complexity rate'. Therefore, this study could reduce the rendering burden on terminals and flexibly deal with the terminal rendering situation by lowering the complexity of the rendering process, thereby providing a smoother and rich media service than existing methods [7].

Thanks to the recent advancements in big data technology, information visualization has been utilized in various data analysis applications [8-9]. In particular, studies on visualization related to image and video data have been actively undertaken [10-13]. These studies provide the researcher or the general user with effective visual information. In particular, they help to understand the distribution of the entire information by abstracting visual information summarized from a huge amount of multimedia data [14], and to effectively find and understand characteristics that cannot be found numerically.

\section{Materials and methods}

This chapter introduces a method of visualizing color information in an image in a 3-dimensional space using standard color models and visualization parameters.

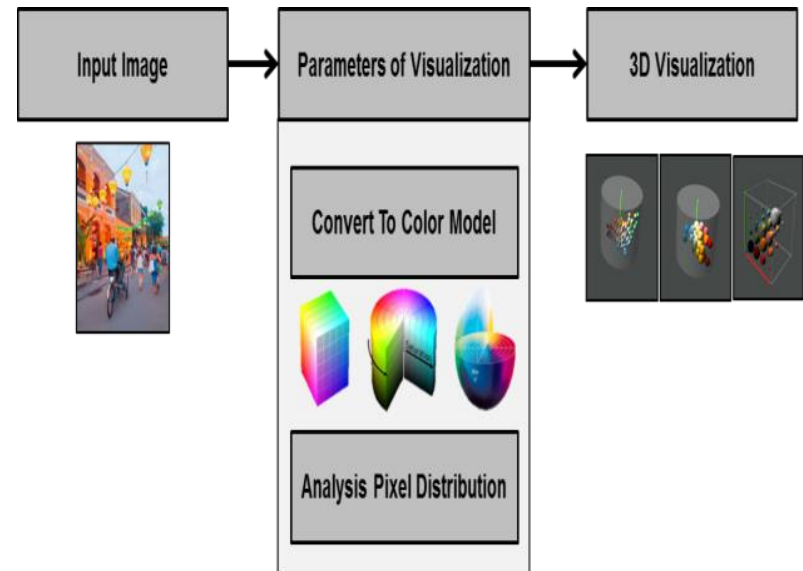

Fig. 2:.System Flow of Color Distribution Visualization.

Figure 2 shows the overall flow of the proposed image color distribution visualization system. The proposed system can control color models and resolutions according to the parameter values defined in Table 1. Each pixel of an input image is analyzed based on the visualization parameters (color model, resolution) set by the user.
The converted color values are then transformed to a 3D object and visualized in a 3-dimensional space.

Table 1: Description of Color Model Visualization Parameters

\begin{tabular}{|c|c|}
\hline $\begin{array}{l}\text { Parame- } \\
\text { ters }\end{array}$ & Description \\
\hline $\mathrm{C}_{\text {model }}$ & $\begin{array}{l}\text { Select the standard color model (RGB, HSV, Lab) for 3D } \\
\text { visualization }\end{array}$ \\
\hline $\mathrm{n}$ & Control the resolution for $3 \mathrm{D}$ visualization \\
\hline
\end{tabular}

\subsection{Visualization based on standard color models}

Color information of pixels of an image should be analyzed to decide the color processing process of an image input by the user. All pixels of the input image have the position information of the RGB color models and can be switched to other color models such as HSV and Lab.
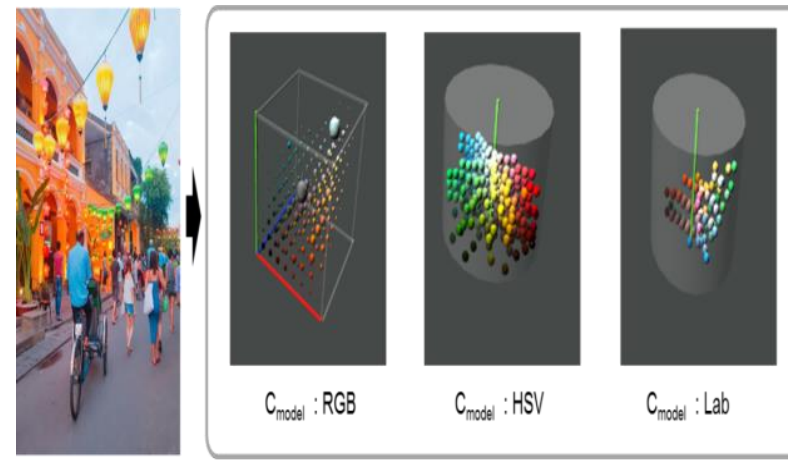

$C_{\text {model }}: R G B$

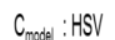

$C_{\text {model }}:$ Lab

Input Image

Fig. 3: 3D Visualization of Color Models for an Input Image

Figure 3 shows the results of visualization by converting an input image into standard color models (RGB, HSV and Lab). First, the $\mathrm{R}, \mathrm{G}$, and $\mathrm{B}$ channels are mapped onto the $\mathrm{X}, \mathrm{Y}$, and $\mathrm{Z}$ axes in the RGB color space. In the HSV color space, the $S$ channel is mapped onto the $\mathrm{X}$-axis, the $\mathrm{V}$ channel is mapped onto the $\mathrm{Y}$-axis, and the $\mathrm{H}$ value is placed in a 3-dimensional space through the rotation of the $\mathrm{Y}$ axis by setting the scale to 360 . In the Lab color space, the $\mathrm{L}$ channel is mapped onto the $\mathrm{Y}$-axis and the $\mathrm{a}$ and $\mathrm{b}$ channels are placed in a 3-dimensional space after calculation.

\subsection{Resolution adjustment}

The resolution of images has been increasing exponentially due to the advancement of technology. This has led to a significant increase in the number of pixels in an image. If all the pixels of an image are expressed in a limited 3-dimensional space, there is a problem that the amount of computation increases and the storage space becomes large. There is also a problem that duplicated color values are continuously processed. Therefore, this section proposes a method for dividing a 3-dimensional space and placing the divided sections into a space and also proposes a method for expressing the size of the pixels placed in each divided space.

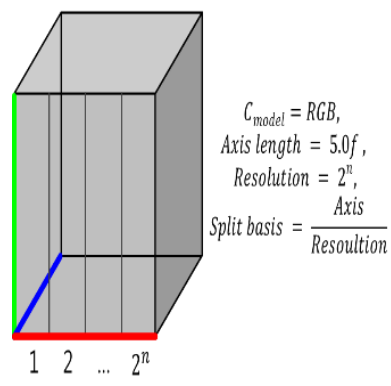

(a) $C_{\text {model }}=R G B$

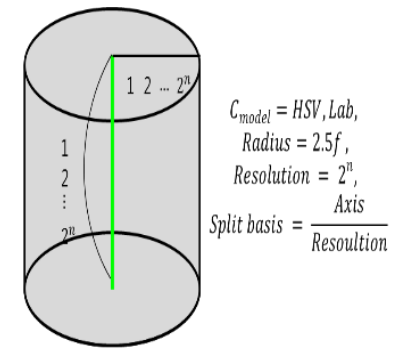

(b) $C_{\text {model }}=H S V$, Lab
Fig. 4: 3D Space Division Model Based on Color Models 
Figure 4 shows that the desired resolution is obtained by dividing a 3-dimensional space into spaces according to the value of the visualization parameter $\mathrm{C}_{\text {model. }}$. In case the value of the $\mathrm{C}_{\text {model }}$ is the RGB color model, and if the axis length is Axis as evaluated from right equations of Figure 4(a) and the resolution can be controlled by $n$, then the space is divided according to the resolution by dividing each axis based on the respective scale. We use the 5.0f value as size of Axis in 3D space coordinates system. In case the value of the $\mathrm{C}_{\text {model }}$ is the HSV or Lab color model, and if the radius of the cylinder is Radius as evaluated in left equations of Figure 4(b) and the resolution also can be Resolution parameter $n$. The space is divided according to the resolution by dividing the radius and height of the cylinder based on the respective scale.

\subsection{Volume size for visualized primitive}

In our visualization system, the number of pixels in an image is expressed in a 3-dimensional space as 3D primitive. We used 3D Sphere object as primitive which represent the number of pixels. If the count of pixels in the same divided area in 3D space, there is a problem that recognition degradation occurs due to size of visualized primitive. Therefore, we adjust the volume size of visualized primitive according to the resolution. In addition, objects are expressed in a 3-dimensional space using a fixed size for the HSV and Lab models.

$$
\begin{aligned}
& \text { Axis }=5 . \mathrm{f}, \text { Resolution }=2^{n} \text {, Scale }=\frac{\text { Axis }}{\text { Resolution }}, \\
& \text { Volume Size }=\frac{\text { count }^{0.32}}{\text { Length }} \times \text { Scale }
\end{aligned}
$$

Equation 1 is example of equation used when the value of Cmodel is RGB. The scale defined above is used for obtaining the size of the $3 \mathrm{D}$ object. The size is determined by dividing the number of counted objects by the number of pixels in an image. If the method defined in Equation 1 is used, regardless of the number of pixels in an image, the size of the objects is appropriately visualized.

\section{Results and discussion}

The follows present the results of the visualization of input images in a 3-dimensional space using the visualization parameters (Cmodel, n) provided by the proposed system. Figure 5 shows a $3-$ dimensional visualization of the distribution of pixels according to the visualization parameter Cmodel and Resolution parameter $\mathrm{n}$ is 2 when the three images are inputted. As you seen in Figure 5, Color distribution is well recognized in 3D color space and user can recognize where the colors are densely packed and where they are less dense.
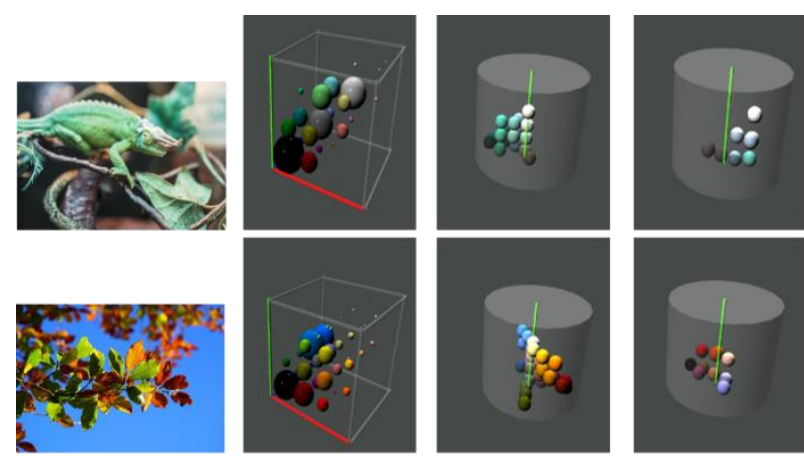

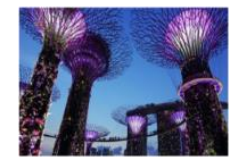

Input Images

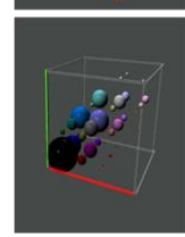

$\mathrm{C}_{\text {model }}=\mathrm{RGB}$

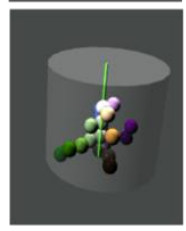

$\mathrm{C}_{\text {model }}=\mathrm{HSV}$

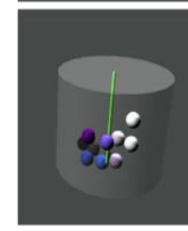

$\mathrm{C}_{\text {model }}=\mathrm{Lab}$
Fig. 5: 3D Visualization of Color Distribution According to Cmode $\mathrm{C}_{1 . .}$

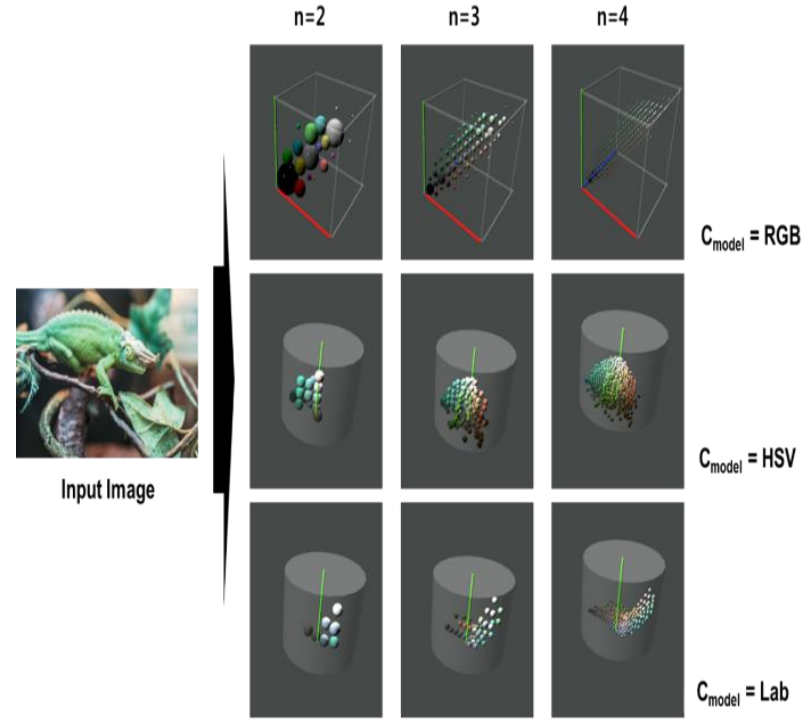

Fig. 6: 3D Visualization of the Color Distribution According to Cmodel and $\mathrm{N}$

Figure 6 shows the visualized results by adjusting the visualization parameters $\mathrm{C}_{\text {model }}$ and $\mathrm{n}$ when inputting the image shown on the left. As the resolution increases, the color distribution appears clearly. On the other hand, only an approximate color distribution is shown as the resolution decreases. Our system can change the style of third visualization by changing the provided visual parameters.

\section{Conclusion}

This study introduced a 3-dimensional method for the visualization of color information and distribution of images. For visualization, a visualization space was designed to understand and visualize the 3-dimensional structure of standard color models (RGB, HSV, and Lab). In addition, it provided not only color models but also resolution parameters to control the accuracy of visualization so as to provide various visualization methods. By expressing the complexity, position, and inter-relationships of color distribution charts in 3-dimensions, we implemented a system that enables the user to intuitively understand and recognize them.

In the future, the results of this research need to be expanded to enable wider use in various image processing applications dealing with colors. For this, we need to consider not only the visualization of the distribution of color information of the currently implemented images, but also how to effectively visualize the analysis results based on the relationship of colors for assessing the color sensibility that has been recently discussed, and using the color clustering technique for the analysis of color sensibility. Lastly, if the system is expanded to support multiple images and videos rather than a single image, it will serve as a color information visualization tool that can be used in more diverse areas.

\section{Acknowledgment}

This research was supported by Basic Science Research Program through the National Research Foundation of Korea (NRF) funded by the Ministry of Education (No. 2016R1D1A1B03935378).

\section{References}

[1] Ware, C. (2012). Information Visualization, Third Edition: Perception for Design (Interactive Technologies) Third Edition, Morgan Kaufman.

[2] Evergreen, S. (2016). Effective Data Visualization: The Right Chart for the Right Data, SAGE Publications Inc.

[3] Gonzalez, R. C., \& Woods, R. E. (2017). Digital Image Processing 4th edition, Pearson. 
[4] Liu, S., Cui, W., Wu, Y., \& Liu, M. (2014). A survey on information visualization: recent advances and challenges. The Visual Computer, 20(12), 1373-1393.

[5] Moreland, K., (2013). A survey of visualization pipelines. IEEE Transactions on Visualization and Computer Graphics, 19(3), 367378.

[6] Chi, E.H.H., (2000). A taxonomy of visualization techniques using the data state reference model. Proceeding of the IEEE Symposium on Information Visualization InforVis, 9-10.

[7] Moere, A.V., Tomitsch, M., Wimmer, C.,Bösch,C., \& Grechenig, T. (2012). Evaluating the effect of style in information visualization. IEEE Transactions on Visualization and Computer Graphics, 18(12), 2739-2748

[8] Geng, Z., Peng, Z., Laramee, R.S., Roberts, J.C., \&Walker, R. (2011) Angular histograms: frequency-based visualizations for large, high dimensional data. IEEE Transactions on Visualization and Computer Graphics, 17(12), 2572-2580.

[9] Wee, M. C. (2017). An improved diversity visualization system for multivariate data. Journal of Visualziation, 20(1), 163-179

[10] Robertson, G., Card, S., \& Mackinlay, J. (1993). Information Visualization Using 3D Interactive Animation. Communications of the ACM, 36(4), 56-71.

[11] Christel, M., Olligschlaeger, A., \& Huang, C. (2000). Interactive maps for a digital video library, IEEE Multimedia, 7(1), 60-67.

[12] Chen, T., Lu, A., \&Hu, S.-M. (2012). Visual storylines: semantic visualization of movie sequence. Computer \& Graphics, 36(4), 241249.

[13] Pretorius, A.J., Bray, M.-A., Carpenter, A.E., \& Ruddle, R.A. (2011) Visualization of parameter space for image analysis. IEEE Transactions on Visualization and Computer Graphics, 17(12), 2402-2411

[14] Colombo, C., Del Bimbo, A., \& Pala, P. (1999). Semantics in visual information retrieval, IEEE Multimedia, 6(3), 38-53. 